\title{
INFECTION \\ PrEParation to be PROUD of
}

The results of the pilot stage of the PROUD study-the first pragmatic open-label randomized controlled trial investigating pre-exposure prophylaxis (PrEP) with tenofovir-emtricitabine for reducing the risk of HIV infection-have been published in The Lancet. PROUD aims to mimic how PrEP would be used in routine clinical practice to allay concerns that its effectiveness would be compromised in a real-world setting.

The pilot study included $544 \mathrm{HIV}$ negative gay men and other men who have sex with men. Participants were randomly assigned 1:1 to receive immediate PrEP or deferred PrEP. The investigators employed procedures that would be likely in a public health PrEP programme. Participants were asked to attend clinic every 3 months for an HIV test, screening for bacterial STIs and testing hepatitis B and C, if indicated. Information on sexual behaviour and adherence to PrEP was gathered via yearly and monthly questionnaires and a daily diary. The primary outcome was time to accrual and retention of participants.
Secondary outcomes included HIV infection, safety, adherence and risk compensation.

In the immediate-PrEP group, 21 men interrupted or missed doses owing to adverse events. Of these events, 13 were considered to be caused by the study drug and were most commonly nausea, headache and arthralgia. Use of postexposure prophylaxis was common in the deferred-PrEP group, with 174 courses being prescribed. Overall, $94 \%$ and $90 \%$ of the expected person years were collected for the immediate-PrEP and deferred-PrEP groups, respectively.

HIV incidence was significantly lower in the immediate-PrEP group than in the deferred-PrEP group $(P=0.0001)$. In a similar population, 13 men would need access to 1 year of PrEP to avert one HIV infection. With regards to STI diagnosis, after adjustment for the number of STI screens, no significant difference was observed between the two groups.

These results refute the concerns that the effectiveness of PrEP would be

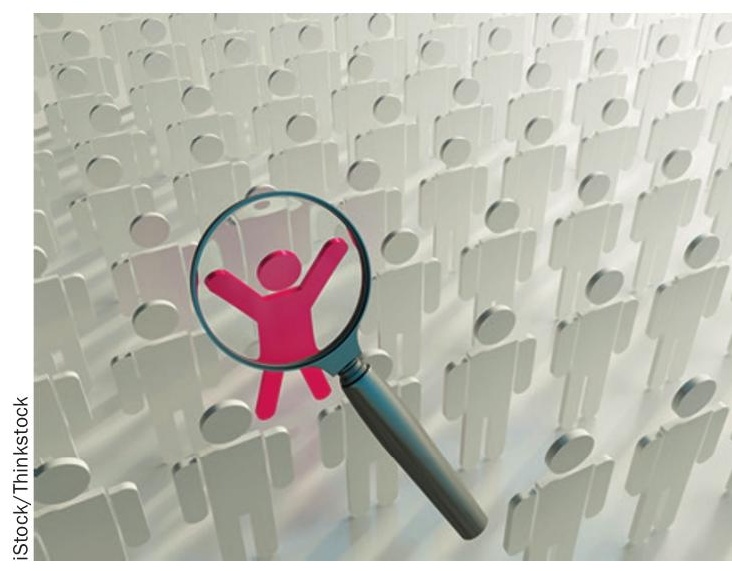

compromised in a real-world setting and would result in risk compensation. A public health programme of PrEP could have a major role in preventing HIV infection and addition to the standard of prevention for men at risk should be considered.

Louise Stone

Original article McCormack, S. et al. Pre-exposure prophylaxis to prevent the acquisition of HIV-1 infection (PROUD): effectiveness results from the pilot phase of a pragmatic open-label randomised trial. Lancet doi:10.1016/S0140-6736(15)00056-2 